\title{
General conclusions
}

\author{
H. Vasey \\ Clinique d'Orthopédie et de Chirurgie de l'Appareil Moteur, Hôpital Cantonal Universitaire, CH-1211 Genève 14, Suisse
}

Substantial progress has been made during the last few years in the treatment of osteo-articular infections.

The particular situation of the bacteria in contact with a foreign body was well demonstrated by Gristina. The production of glycocalix by microorganisms as well as biofilms (fibronectine, fibrin, laminin and others) left on implants alter the influence of antimicrobial agents. Surgical treatment remains the key to success. It aims at eliminating the devitalized and infected tissues, reducing the number of bacteria and improving the perfusion of infected zones. In this respect, a recent major contribution is the more frequent and early use of free or pedicle muscle flaps, to fill the bone defects and bring a vascular bed to the infected and scarred zones.

The Ilizarov technique of osseous transport solves the problems of loss of cutaneous as well as osseous substance. The use of "spacers", bullets or blocks, of cement, generally impregnated with antibiotics, allows insertion of new total prostheses some time after their removal.

The local supply of antibiotics obeys the logic of carrying the substance to where it is usefull. Administration by internal pump (Perry 1989) or external pump (Meani 1993) opens up new ways for long lasting treatments. New media are available : collagen (Braun 1985) with its new forms, fibrin, cancellous bone, methyl polymethacrylate treated in order to change the distribution of antibiotics (under vacuum polymerization, with dextran added), biodegradable cement (Gerhardt 1988).

Antiseptics or similar substances are lagging behind. The taurolidin proposed by Pfirrmann in 1985 has been tried and tested by limited groups of users but is not available in numerous countries and, thus, experiences the consequences of a too limited circulation. In 1989 Baltzer published the results of "GX" a biguanid studied among others by Willenegger ${ }^{1}$. This substance is similar to chlorhexidine and seems to me to have some future

\footnotetext{
${ }^{1} \mathrm{GX}$ is marketed under the name "Lavasept"

because of the absence of toxicity and a very large spectrum of action. Bacterial resistance to this substance is not known.

In the current context of development of resistance to antibiotics as they are introduced, one can only hope for the arrival of an effective local chemotherapy.

To conclude, the major problems we have to face seem to be the following:

- Septic arthritis still depends on systemic antibiotic treatment. Local antibiotics cannot be prescribed in high dose because of their resorbtion, antiseptics are toxic to cartilage. The cases seen still end up late with important functional sequelae.

- Colonies of methicillin-resistant staphylococci become a worrying problem of hospital hygiene in departments of septic orthopaedics.

- Some mycobacteria responsible for osteitis are resistant to known tuberculostatics. The duration of antibiotic treatment, of bone infections, remains arbitrary. The length of intravenous administration, often for six weeks, requires expensive hospitalizations. We are lacking the objective parameters which could enable us to end treatment. 